\title{
Automatic fall monitoring using floor vibration
}

\author{
Marco Tarabini ${ }^{1}$, Filip Gocanin ${ }^{1}$, Bortolino Saggin ${ }^{1}$, Diego Scaccabarozzi ${ }^{1}$, Marco Bocciolone ${ }^{1}$ \\ ${ }_{1}^{1}$ Politecnico di Milano, Department of Mechanical Engineering, Via La Masa 1, Milan, Italy
}

\begin{abstract}
This work investigates the possibility of monitoring the activity and the falls of people in dwellings using three or more accelerometers fixed on the ground. The main difference between the proposed method and existing ones is the use of acceleration to estimate the impact force by using the apparent mass of the floor; the latter is experimentally identified in each room in which the tests were performed using the heel drop test. The study has two parts: 1 . the apparent masses of different dwellings' floors have been measured. 2. the ground reaction force is studied using a purposely designed force platform with a surface of approximately $2 \mathrm{~m} \times 1 \mathrm{~m}$. The force platform allowed the measurement of the forces generated by the falls of 21 subjects, of a crash test dummy (falling in front or rear direction from seated and standing position, with or without the interposition of objects on the trajectory), and of common objects (e.g. dishes, water bottles, books). The impact location is estimated by triangulation, using a wavelet algorithm derived from the existent literature. The results show the possibility of identifying the presence of subjects inside the room and the fall of subjects in the majority of dwellings. We conclude that the proposed method allows a clear distinction between the fall of subjects and objects, given that the difference in terms of force (which is estimated from the floor's apparent mass and from the measured acceleration) is at least of one order of magnitude.
\end{abstract}

\section{Section: RESEARCH PAPER}

Keywords: vibration, fall monitoring, measurements, assisted living, active ageing

Citation: Marco Tarabini, Filip Gocanin, Bortolino Saggin, Diego Scaccabarozzi, Marco Bocciolone, Automatic fall monitoring using floor vibration, Acta IMEKO, vol. 8, no. 1, article 7, March 2019, identifier: IMEKO-ACTA-08 (2019)-01-07

Section Editor: Ronaldo Dias, INMETRO, Brazil

Received October 01, 2017; In final form January 15, 2019; Published March 2019

Copyright: (C) 2019 IMEKO. This is an open-access article distributed under the terms of the Creative Commons Attribution 3.0 License, which permits unrestricted use, distribution, and reproduction in any medium, provided the original author and source are credited.

Funding: This work was supported by a grant from Regione Lombardia ('Avviso pubblico per la realizzazione di progetti di ricerca industriale e sviluppo sperimentale nel settore delle Smart cities and Communities - POR-FESR 2007-2013 asse 1 - Linea di intervento 1.1.1.1. azione E').

Corresponding author: Marco Tarabini, e-mail: marco.tarabini@polimi.it

\section{INTRODUCTION}

The continuous improvement of living standards has created new challenges for humanity: the average extended human lifespan is one of the major burdens on today's healthcare systems. Estimations show that by 2050, we could expect the number of elderly persons living in their own homes but requiring assistance to triple [1]. Falls are one of the most hazardous scenarios [1], [2]. Approximately $33 \%$ (range: $15 \%$ to $44.9 \%$ ) of elderly community-dwelling persons in the USA are older than 65 years, and up to $60 \%$ of nursing home residents fall each year [3]-[5]. Falls (loss of balance resulting in coming to rest on the floor) are the one of the major causes of injuries resulting in death and of injury-related hospitalization among senior citizens.

Different works have focused on the possibility of detecting a fall using portable systems [5]-[24]. The majority of the methods rely on wearable sensors [7]-[10] and vision techniques [11]-[18]. The main limitation of the wearable systems is that these methods are not effective when the sensor is not worn, and elders show a certain hostility versus new technologies and often forget to wear or recharge the sensor. Vision systems might fail in the presence of occlusions between the camera and the subject; consequently, the experimental setup might be complex if it is necessary to cover the entire house surface. The use of floor vibration as a diagnosis for the presence or fall of people inside a room has already been considered in the literature [19][19]. Alwan et al. [19] observed that, as human activities cause measurable vibrations on the floor [25], it is possible to detect human falls by monitoring vibration patterns. The above hypothesis entails that the vibration signature of the floor differs from that of common daily life activities and from that of falling objects. The authors used piezoelectric accelerometers preloaded against the ground using a mass and spring system. The system includes battery-powered pre-processing electronics that start from the measured vibration, which produce a binary fall signal. We performed the tests in a controlled laboratory environment and concluded that it is possible to detect falls by using vibration 
patterns. The detection range was around $4.5 \mathrm{~m}$ on concrete slab floors. We consider that the results depend on the floor dynamics, which can be measured with the instrumented heel drop test proposed by Blakeborough and Williams [25], but no further details are reported in the paper. In the standard heel drop test, a subject with mass standing on the balls of his feet, with talons at approximately $8 \mathrm{~cm}$ from the ground, suddenly relaxes and lets the heels fall on the ground.

Litvak et al. [20] and Ziegel et al. [21] proposed an automatic fall detection system based on floor vibration and acoustic noise. A pattern recognition algorithm was used to discriminate between falls and spurious events. Tests for human falls were simulated using a mannequin and were only performed in a laboratory environment; to date, no study has compared the force of dummies and of young subjects. Furthermore, the forces generated by older adults may be different from those of young subjects because of the elderly persons' limited muscular force and their reduced mental alertness. The works of Alwan, Allen, and Ziegel are highly relevant in the field, but they did not analyse vibration transmissibility in residential buildings, nor did they consider that falls against objects or from seated positions may result in very low acceleration levels.

In this work, we outline our study for the detection of people's falls using an estimation of ground reaction force (GRF). The estimation is performed by measuring floor vibration by accelerometers attached to the floor and by characterising the floor's apparent mass and the vibration transmissibility before starting the measurements. Preliminary tests for the discrimination between falls of objects and subjects were performed by using a purposely designed force platform. In addition to the validation of the method, this paper investigates:

- the transmissibility of vibrations in different residential floors that have not yet been studied. Given that a fall generally occurs at variable distances from the sensors, it is necessary to identify the vibration transmissibility in order to assess building-dependent modifications of the signal;

- the force generated by people fall is studied for the frontal and rear falls of young healthy subjects [27]-[29];

The paper is structured as follows: the proposed method is described in section 2. Section 3 describes the experiments performed in the laboratory (falls of a crash test dummy, athletes, and objects), in dwellings (identification of vibration transmissibility), and in real conditions. Results are discussed in section 4, and the study's conclusions are presented in section 5 .

\section{MATERIALS AND METHODS}

The detection of subjects' falls using the floor vibration measured on the ground is influenced by three phenomena: the vibration is modified by the floor transmissibility, the force generated by the impact is unknown, and there may be other events leading to vibration signals that might be similar to that deriving from a person's fall.

The proposed approach is presented in Figure 1: the vibration transmissibility through the different grounds and the force generated from the different impacts are studied separately in order to be able to predict the vibration generated by different falls on the different floors. In other words, with the separate characterization of the force generated by an impact on an infinitely rigid floor and of the vibration transmissibility through the floor, it will be possible to understand a variety of combinations between impact locations, fall types, and floor characteristics. The estimated force signals will be used in future

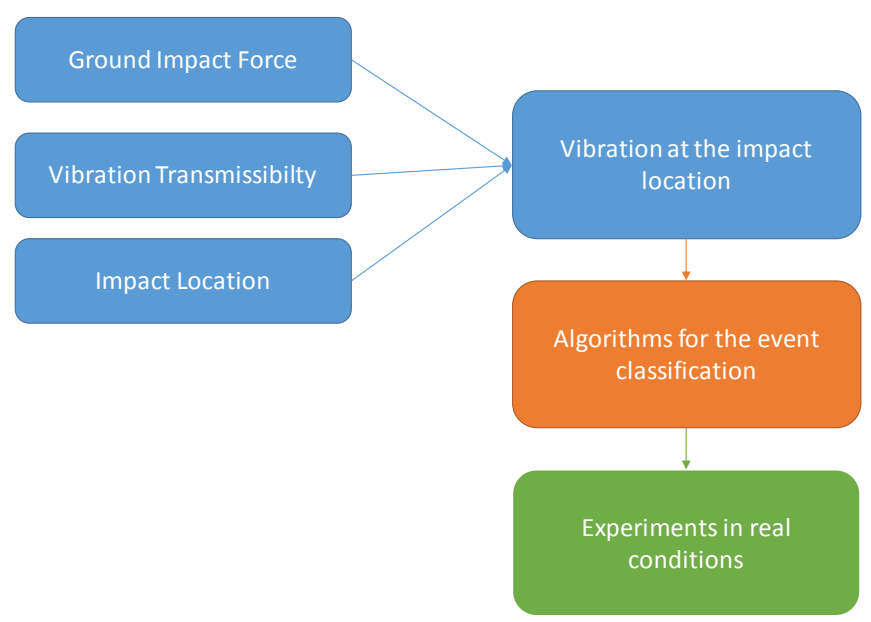

Figure 1. Schematic of the proposed method.

works as training phases for the algorithms for event detection, similar to [30]-[32].

\subsection{Proposed method}

The dependence between the fall impact force and the measured floor vibration is constrained by the ground mechanical impedance, i.e. the ratio between the force and the velocity, or by the ground apparent mass, i.e. the ratio between the force and the acceleration [33]-[36]. If the behaviour of the ground is linear, both the apparent mass and the impedance can be estimated with impact tests similar to those described in [26]. The ground apparent mass $(A M)$ can therefore be estimated at low frequencies with the heel drop test by knowing the force generated by the heel $(F)$ and the acceleration $(a)$ measured at position $j$ when the heel drop test is performed at location $i$.

$A M_{i, j}(f)=\frac{F_{i}(f)}{a_{j}(f)}$

Given that this quantity is constant (if the ground behaviour is linear), if the subject falls close to location $i$, it is possible to estimate the force generated by the fall by multiplying the apparent mass of the ground $A M_{i j}(f)$ times the measured acceleration during the real fall at position $j$.

$F_{i, \text { fall }}(f)=A M_{i, j}(f) \cdot a_{j, \text { fall }}(f)$

With this method, it is possible to classify the fall events using the force instead of the acceleration as used in all the existing methods required by existing studies. The proposed approach requires knowledge of the impact location, which in this case, was identified using the wavelet method described in [37]. The method requires positioning at least three triaxial accelerometers on the ground in order to detect the plate longitudinal waves. The continuous wavelet transform, using the Gabor function as mother wavelet as suggested in [37], was used to identify the time of arrival of the waves to the different sensors. At each frequency, the time difference between the first arrivals to two sensors is related to the so-called wave group velocity through the known distance between two sensors. The group velocity $C_{g}(f)$ was therefore computed as the ratio between the distance $L$ and the difference between the times of arrival $t_{1}(f)$ and $t_{2}(f)$ computed from the wavelet scalogram at frequency $f$.

$C_{g}(f)=\frac{L}{t_{2}(f)-t_{1}(f)}$

In this study, the time of arrival was evaluated by analysing only the fastest propagation mode identified by the scalogram maxima. 
Once the location of the impact was identified, the force time history could finally be computed using the inverse Fourier transform of equation (2).

\subsection{Ground transmissibility}

The impact on the ground generates compressive and flexural waves that propagate through the floor from the impact position to the sensors' location. The subjects' fall can be identified by observing different features of the signal (in either the time or frequency domain) and by using information about the impact location (to discard, for instance, shocks occurring in specific positions). The first step for the feasibility study is the identification of the floor vibration transmissibility in residential buildings given that if the transmissibility tends to zero, it is not possible to also measure the vibration at small distances from the impact location. Therefore, we decided to analyse a group of houses with different characteristics in order to evaluate the possibility of locating the transducers in positions that are not critical for the daily activities of people.

Since the vibration transmissibility depends on the mechanical and geometrical characteristics of the base and of the floor, experiments were performed in different conditions. For the purposes of this study, four IEPE accelerometers model Bruel\&Kjaer 4508 B, with nominal sensitivity of $10 \mathrm{mV} /\left(\mathrm{m} / \mathrm{s}^{2}\right)$ measured the vibration at the positions indicated in Figure 2.

Three accelerometers (indicated by green circles in Figure 2) were fixed at positions that depended on the room dimensions (longer room side $a$, shorter room side $b$ ). Another accelerometer was moved close to the impact location. The vibration signals were sampled using a National Instruments NI 9234 data acquisition board. The sampling frequency was $2048 \mathrm{~Hz}$. The stimulus was given by the force generated in a heel drop test. The subject that performed these tests also performed the same tests on the instrumented platform described in section 2.3 (20 tests performed in repeatability conditions), in order to obtain the average excitation force together with its variability (the standard uncertainty was $13 \%$ in our tests).

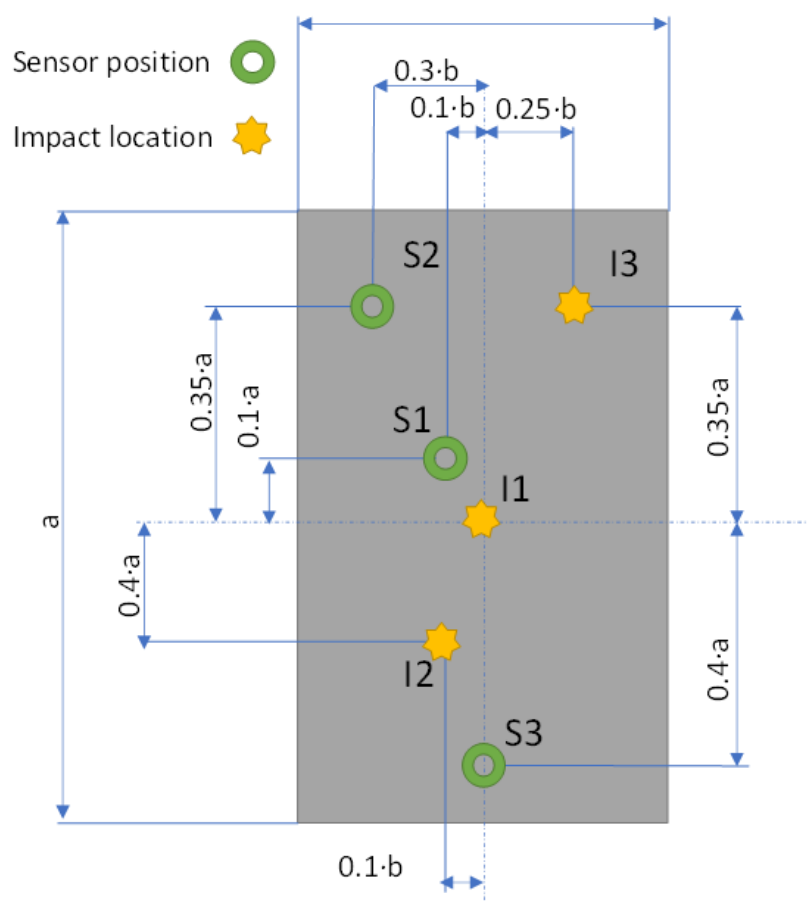

Figure 2. Position of the accelerometers and of the impact in the transmissibility tests.
The vibration transmissibility and the ground apparent mass were measured in 40 rooms, with surfaces between $2 \mathrm{~m}^{2}$ and 50 $\mathrm{m}^{2}$, with different floor materials (wood, stones, tiles). The experimental results have been summarised by averaging the vibration transmissibility of different buildings. The latter was measured using the $\mathrm{H} 1$ estimator of the frequency response function, by averaging the results of five tests (lasting $2 \mathrm{~s}$ with a pre-trigger of $0.3 \mathrm{~s}$ ). The ordinary coherence function has been computed as well.

\subsection{Measurement of the ground reaction force}

Given that the proposed method is based on the computation of the GRF, we have designed a force platform with a surface large enough to perform fall tests. The platform was built with a sandwich honeycomb panel $(2.5 \mathrm{~m} \times 1.25 \mathrm{~m})$ supported by four piezoelectric load cells PCB 211B. The sandwich thickness is $100 \mathrm{~mm}$, with a sheet thickness of $1 \mathrm{~mm}$ and a honeycomb thickness of $50 \mu \mathrm{m}$. The upper sandwich layer was covered by $5 \mathrm{~mm}$-thick compensated wood in order to protect the surface from the localised impacts generated by the dummy. The theoretical computations pointed out a resonance frequency of approximately $85 \mathrm{~Hz}$. The dynamic behaviour of the force platform has been experimentally verified with an impact hammer, and we found that the frequency pass-band $( \pm 3 \mathrm{~dB})$ was $40 \mathrm{~Hz}$. The first natural frequency of the unloaded plate was $63 \mathrm{~Hz}$. Furthermore, in this case, data was pre-triggered so that the first impact of the object on the platform occurred after $0.3 \mathrm{~s}$.

Three groups of simulations were performed:

- Fall simulations performed by subjects falling forward and backward with complete fall arrest: tests were performed on the force platform by 21 healthy young subjects with a height between $1.65 \mathrm{~m}$ and $2.00 \mathrm{~m}$ and a body mass between $45 \mathrm{~kg}$ and $95 \mathrm{~kg}$ (average $72 \mathrm{~kg}$ ). Subjects were instructed on how to perform the simulation to avoid any injuries. Tests were performed in accordance with the ethical guidelines of Politecnico di Milano. The tests differed because of:

- Direction of fall: front fall (F) or rear fall (R)

- $\quad$ Subject body mass (in kg)

- $\quad$ Subject height (in m)

- Hip height (in m)

- A fall simulation performed by a dummy (Humanetics pedestrian dummy, Hybrid III 50th Percentile, mass $104 \mathrm{~kg}$ ) was used to identify the force generated by different fall configurations. Simulations were performed with and without limited fall arrest according to the following configurations:

- Type of fall: rear, front, or side

- Pre-fall posture: standing or sitting

- Height of the hips before fall: $0.5,0.6,0.7$, or $0.8 \mathrm{~m}$

- Limbs arrest posture: no arrest, one arm arrest, two arms arrest, and elbow arrest

- Distance between the feet before the fall: 0.2 or $0.3 \mathrm{~m}$

- Description of fall trajectory: free trajectory or fall over the objects

- Falls of common objects of different weights, sizes, and shapes from different heights:

- Objects: plastic bottle, glass, glass bottle, dish, pot or box

- Object weight

- Height of the fall: 0.7 or $1.4 \mathrm{~m}$

- Number of falling objects 
During the experiments, both the acceleration and the GRFs were recorded. Data was summarised using basic descriptive statistics. The ratio between the average mass of the subjects that performed the test and the mass of the dummy is 0.7 , and the GRF should also be in a similar proportion if the fall configuration is similar.

Not all the possible combinations between factors were included given that parts of them were meaningless: for instance, it is almost impossible in a frontal fall to have an elbow impact. Only realistic fall configurations were reproduced and analysed.

\subsection{Method validation}

The proposed method for the localisation of the fall using wavelets and for the computation of the force is validated in two steps. The first validation was performed on the force platform: the expected vibration of the sensor was computed using equation (2) using the location of the impact (estimated using the wavelet triangulation) and the force measured by the load cells. The expected vibration has been compared with the acceleration measured at different locations. The difference between the estimated and the measured acceleration of all the tests was used in order to assess the method's reliability.

In the second step, an embedded system, based on MEMS accelerometers and a Raspberry PI board, was installed in a residential building, and the vibration was monitored inside a small bath and in the bedroom. The rooms were characterised using the method described in section 2.1. The tests aimed for the validation of the architecture and for detecting common activities of daily living.

\section{RESULTS}

\subsection{Method validation}

The method was validated by comparing the acceleration measured on the force platform, predicted using the measured GRF and the apparent mass of the force platform itself. Figure 3 shows an example of the comparison in one of the tests performed by with the crash test dummy.

The results, apart from a tonal component at the resonant frequency of the plate was not reduced by the digital filtration of data, show maximum errors that are lower than $20 \%$. Due to
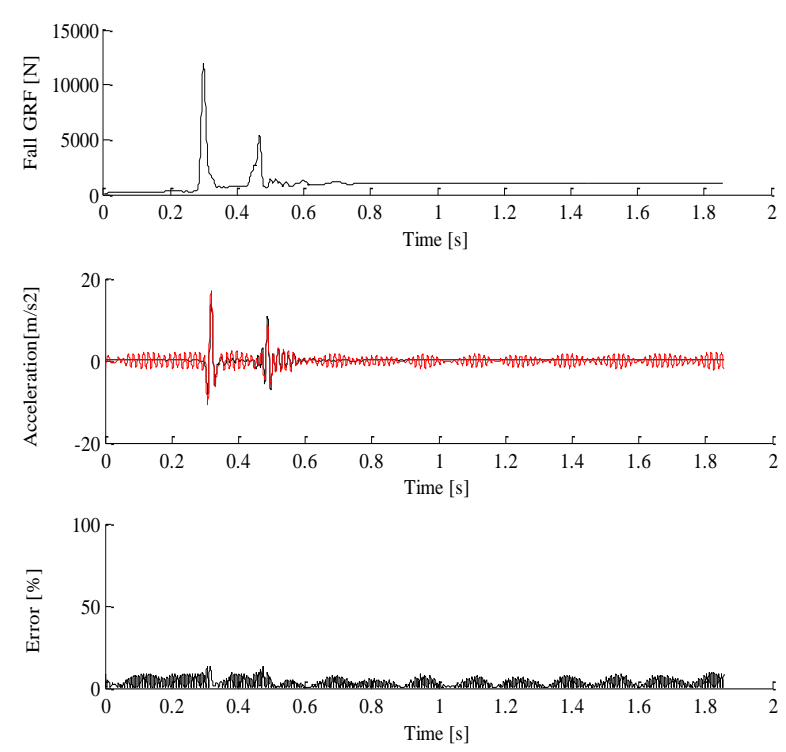

Figure 3. Force signal (upper plot), measured vibration (black line), expected vibrations (red line) (middle plot), and the test error (lower plot).

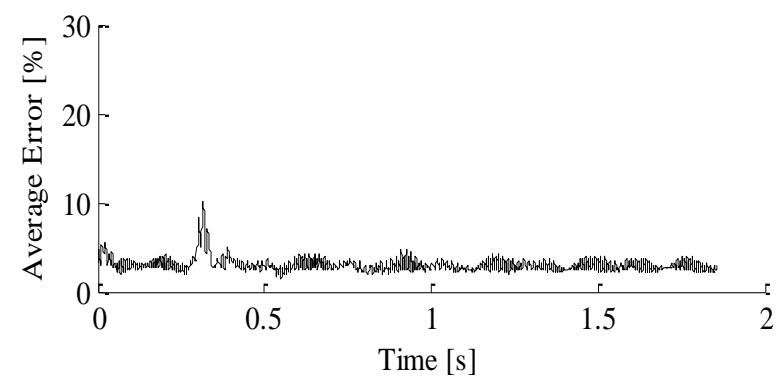

Figure 4. Average difference between the measured and the predicted acceleration generated by the falls of the dummy and subjects.

system linearity, the error is the same for the estimation of acceleration during the method validation (by finding the GRF) or for the estimation of the force during the real tests (by measuring the acceleration and the floor characteristics).

The average error on the amplitude of the first acceleration peak is $23 \%$; the average was computed on the entire data set. The error is due to difficulties in the identification of the location of the impact, which in approximately $50 \%$ of cases resulted in the choice of a point close to the one at which the impact occurred. In addition, the apparent mass of the plate was measured without the mass of the dummy/subject, thus leading to a biased compensation of the FRF. This aspect was confirmed by the spectral analysis of the error, which was dominated by components at frequencies between $55 \mathrm{~Hz}$ and $63 \mathrm{~Hz}$. These values are close to the first natural frequency of the platform and vary between different tests depending on the position of the subject/object.

The average error (Figure 4) decreased to $16 \%$ when the accelerations were computed for the falls of the subjects and the crash test dummy (i.e. excluding the objects, for which the measured forces were characterised by a poor SNR). In these conditions, the error of magnitude of the largest acceleration/force peak decreased to $14 \%$; the average error, apart from the force peak, was lower than $5 \%$. Furthermore, in this case, the spectral analysis evidenced the dominance of the frequency components close to the first resonance of the platform.

\subsection{Ground transmissibility}

The average vibration transmissibility measured in 40 rooms is shown in Figure 5(a), and the average coherence between the input and output position (asterisks and circles in Figure 2) is shown in Figure 5(b). Plots include the effect of the different room sizes, of the floor mechanical characteristics, and of the different positions of the impact and of the sensors.

The plots show that the modulus of the vibration transmissibility is, on average, lower than 1 in the band between $0 \mathrm{~Hz}$ and $150 \mathrm{~Hz}$. The average transmissibility has a minimum below $15 \mathrm{~Hz}$, but the lower value (0.3) does not prevent measurements in that region. The coherence is, on average, larger: between $20 \mathrm{~Hz}$ and $50 \mathrm{~Hz}$. This interval represents that in which the SISO system approximation is more reasonable. At lower frequencies, the effect of non-measured inputs (such as the vibration of the building induced by natural agents or by traffic) might be relevant. Above $50 \mathrm{~Hz}$, the lack of energy in the stimulus might lead to a low signal-to-noise ratio. Further analyses evidenced that the vibration transmissibility and the coherence function depend on the floor type and on the room size: the transmissibility measured on the wooden floors is, on average, lower than that on the tiles and stones, especially at high 

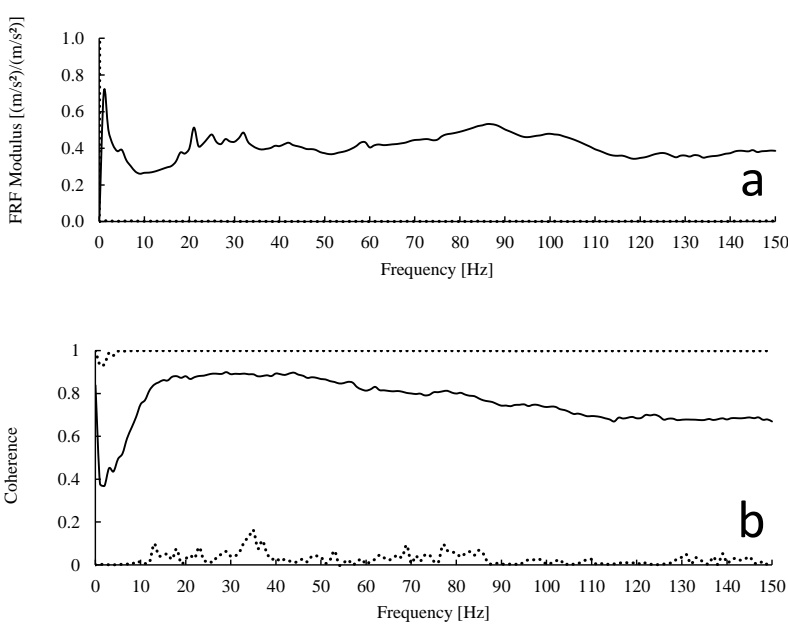

Figure 5. Average transmissibility of the room in which the tests were carried out (a) average coherence of the transmissibility tests; (b) solid line: test average; dotted line: maxima and minima measured during the tests.

frequencies. As expected, the first resonance frequency depends on the room size, while the effect of the furniture is negligible in comparison with the effects of floor type and room size. Three examples of the effects of the room properties on the coherence and transmissibility are shown in Figure 6, Figure 7, and Figure 8.

Figure 6 shows that the transmissibility of the vibration with the parquet is smaller, on average, than the vibration transmissibility when the floor is covered with tiles. The average transmissibility between $1 \mathrm{~Hz}$ and $150 \mathrm{~Hz}$ with the tiles was 0.54 ; the value dropped to 0.26 when the floor was covered by the parquet.

A similar effect was noticed for the room size, where the vibration transmissibility of small rooms $(0.55$ between $1 \mathrm{~Hz}$ and $150 \mathrm{~Hz})$ was, on average, larger than that of large rooms $(0.38$ in the same frequency range). The coherence between the stimulus (force) and the response (acceleration), shown in Figure 7, was independent from the room size, between $15 \mathrm{~Hz}$ and $50 \mathrm{~Hz}$ $(0.87,0.89$, and 0.87 for large, medium and small rooms respectively). At higher frequencies $(100 \mathrm{~Hz}$ to $150 \mathrm{~Hz})$, the coherence measured in large rooms (0.61) was smaller than that of medium (0.70) and small rooms (0.79) because of the lower signal-to-noise ratio when the accelerometer was far from the impact location.

The effect of the ratio between the room dimensions was small on both the transmissibility and coherence (Figure 8 ). The average coherence at frequencies between $15 \mathrm{~Hz}$ and $50 \mathrm{~Hz}$ was 0.88 independently from the ratio between the room dimensions

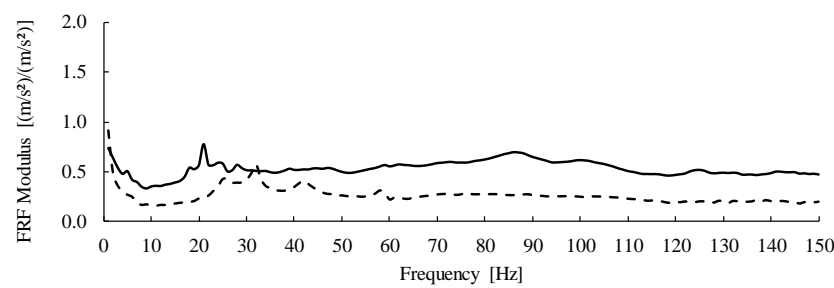

Figure 6. Influence of floor covers on signal transmissibility: floors covered by tiles (solid line) and by parquet (dashed line).

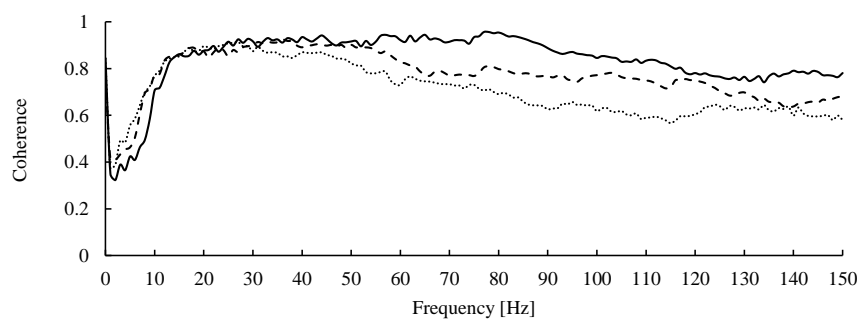

Figure 7. Influence of room size on signal coherence: small-sized rooms (solid line), middle-sized rooms (dashed line), and large-sized rooms (dotted line).

$a$ and $b$. Between $100 \mathrm{~Hz}$ and $150 \mathrm{~Hz}$, the average coherence was 0.76 for rooms with a side ratio that was lower than 2 and 0.84 for rooms with a ratio higher than 2 .

\subsection{Impact force}

The average GRF measured during the fall of the dummy, of the healthy subjects, and of the objects is shown in Figure 9.

The results evidence that the force generated by the dummy is, on average, much larger than that generated by objects and healthy subjects. This is partially due to the larger mass of the Humanetics dummy in comparison with the average subjects' masses. The ratio between maximum force generated (on average) by the fall of a dummy (close to $10 \mathrm{kN}$ ) and the maximum force generated by the fall of a subject (approximately $1 \mathrm{kN}$ ) is 10 , i.e. much larger than the ratio between the masses (1.4). This large difference can be explained by two factors: the first is that the dummy does not have any conscious reaction to the fall. This aspect is typical of elders, who usually do not protect themselves using their arms. The second factor is the difference between the biomechanical characteristics of the crash test dummy and the subjects. This factor is expected to be limited given that the dummy is designed to mimic the behaviour of subjects exposed to impulsive accelerations.

A comparison between the average GRFs and the average accelerations generated by the falls of objects, subjects, and dummies is shown in Figure 10.

The frequency content of the fall of subjects and objects is usually different, as shown by the wavelet transforms in Figure 11. The plot shows that the impact of hard objects (b) creates vibrations at higher frequencies (over $70 \mathrm{~Hz}$ ) while the bandwidth of acceleration generated by the impact of a soft object (paper box, a) is lower than $35 \mathrm{~Hz}$. As a comparison, the spectrum measured during the fall of the dummy is reported in Figure 11(c).

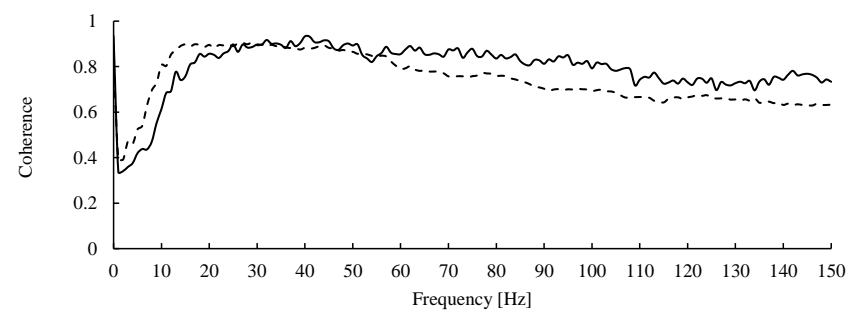

Figure 8. Influence of room dimensions ratio $a / b$ on measured signal coherence: if ratio $a / b$ is higher than 2 (solid line), if ratio $a / b$ is lower than 2 (dashed line). 

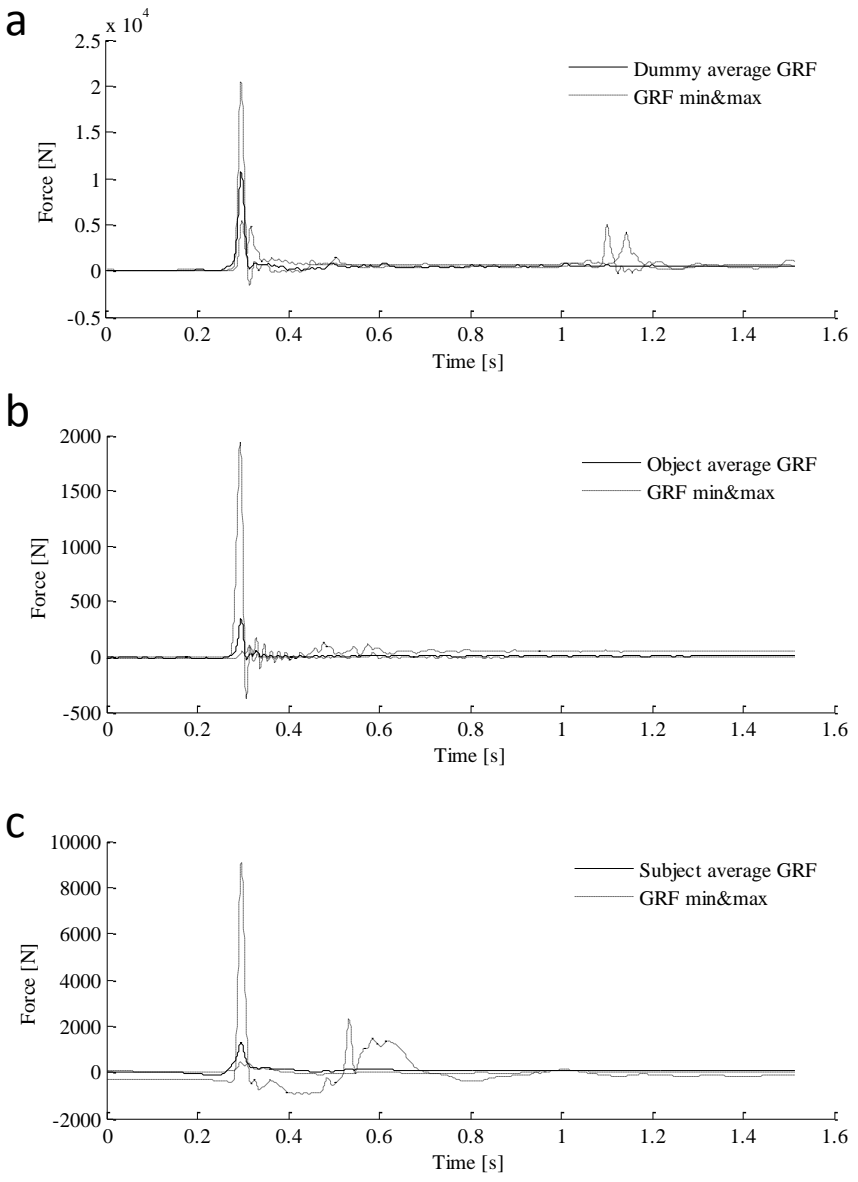

Figure 9. Time histories of the GRF measured during (a) the dummy falls; (b) object falls; and (c) healthy subject falls. Solid lines: averages, dotted lines: maximum and minimum.

The possibility of distinguishing between the falls of objects and people is confirmed by the wavelet transforms of the average GRF measured during all the tests (shown in Figure 12).
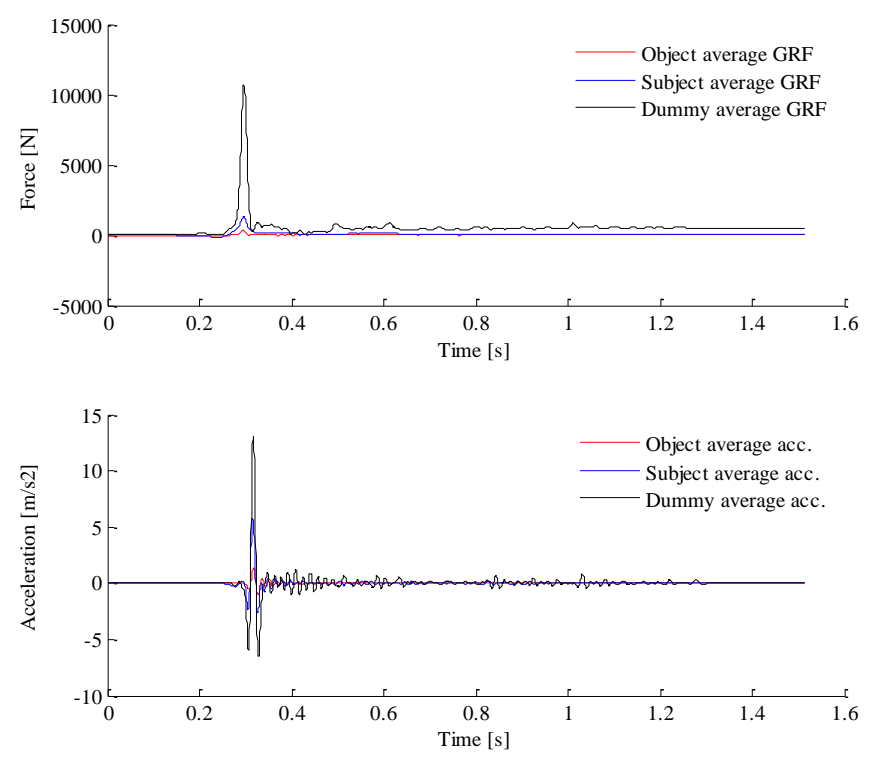

Figure 10. Comparison between the average GRF and acceleration signals obtained during dummy, subject, and object falls.
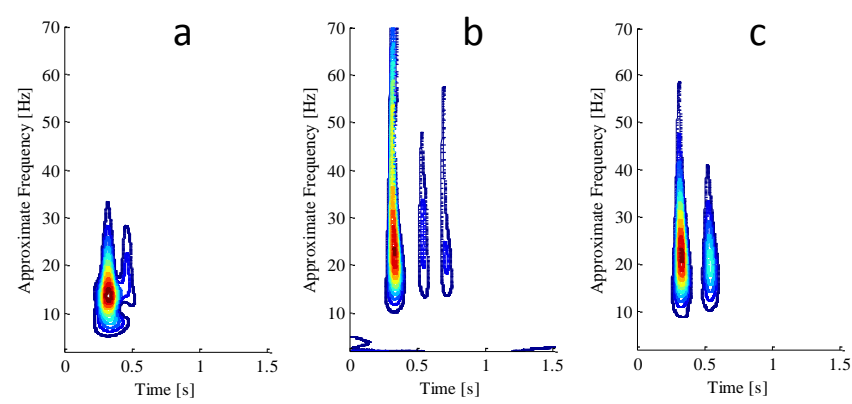

Figure 11. Wavelet transforms of acceleration signals generated by falls of (a) a soft object, (b) a hard object, and (c) the crash test dummy.

Different to the falls of objects, the GRF has relevant components that are also at low frequencies. The dominating frequency components of observed during the falls of objects is close to $15 \mathrm{~Hz}$, while the dominating frequency components of falls generated by subjects are typically below $5 \mathrm{~Hz}$.

Another characteristic that allows for distinguishing the falls of objects from those of subjects is the number of impacts. The falls of both subjects (b) and the dummy (c) usually generate two spectral peaks (the impact of two body parts, such as knees and hands or elbows and head) while falls of objects usually generate a single spectral peak.

\subsection{Preliminary experimental results}

The last step in the research is the installation of the embedded measurement system in a residential building. To date, there have been no falls, and consequently, it has not been possible to validate the fall detection system or to obtain real fall data. Nevertheless, the results of this study have shown that the system detects activities of daily living, showing vibration levels much larger than floor noise during the day.

\section{DISCUSSION}

The results presented in this paper evidence the compatibility between the frequency of fall-generated excitations and the frequency region in which the vibration transmissibility is high. The vibration transmissibility suggests that it is possible to measure the vibration at any position of the room, independent of the impact location; therefore, theoretically, one accelerometer is enough for detecting the presence of people in the room or their fall. The simultaneous use of at least three transducers, however, allows for identifying the location of the fall and consequently for the estimation of the GRF that has generated the vibration. This parameter is, in principle, more reliable than the vibration generated by the fall, which depends on both the event that has generated the fall and on the distance between the impact location and the transducer.
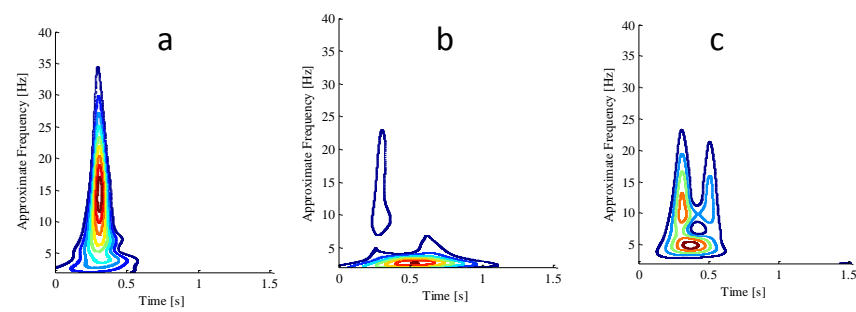

Figure 12. Wavelet transforms of the average of the (a) objects' GRF; (b) subjects' GRF; and (c) dummy's GRF. 
The uncertainty of the GRF is limited by the heel drop test used for the identification of the ground apparent mass. From this perspective, we have already performed tests by dropping a silicone ball from a constant height; the results are certainly repeatable and will be presented in future studies.

The force generated by falls of subjects is larger than that generated by falls of objects, and the identification of a threshold is, in principle, easier than the identification of the threshold for the acceleration level, which depends both on the impact magnitude and on the position of the fall with respect to the accelerometer. The identification of thresholds has not been included in this work and deserves attention in forthcoming studies, in which we will try to use the techniques proposed in [23][32], using the force instead of the acceleration as the main feature for the recognition.

The results show that the use of three accelerometers allow for estimating the location of the fall; however, in approximately $50 \%$ of laboratory falls, the impact location was identified at a point that was adjacent to the actual one (the error being lower than $1 \mathrm{~m}$ ). The optimal transducer location will be studied in forthcoming studies, but thanks to the low cost of the MEMS accelerometers, it seems reasonable to put more than three sensors in each room in order to obtain a more reliable impact position estimation.

The main limitation of this work is surely the lack of experimental validation in real conditions. During the scheduled experimental activities in the residential buildings for elders, there were no falls, and consequently, it was not possible to verify the proposed method. Nevertheless, the results of the tests performed in laboratory conditions were promising, and we expect to obtain more data in future experimental sessions.

\section{CONCLUSION}

In this paper, we have described a method for the detection of human falls in dwellings using GRF as a main parameter for the event classification. The method is based on the identification of the floor's apparent mass using the heel drop test and on the computation of the impact location using at least three triaxial accelerometers. The method was validated on a purposely-designed force platform. Fall simulation tests were performed with a crash test dummy and young healthy subjects. The force generated by falls of objects was also measured as a term of comparison. Given that there is an order of magnitude between the GRF generated by the falls of objects and subjects, the parameter should be more reliable than the acceleration.

Tests were also performed to identify the apparent mass and vibration transmissibility of common residential buildings. The results evidence that the transmissibility is larger than 0.3 up to $150 \mathrm{~Hz}$, thus showing that the vibration generated by the fall (which has a bandwidth close to $40 \mathrm{~Hz}$ ) can be transmitted inside the room and can therefore be measured by the different accelerometers.

Preliminary tests were not useful for the method validation, given that there were no real falls during the measurements. Future developments in this area will include a new series of tests in residential buildings, a more reliable method for the measurement of the ground apparent mass, and the simultaneous usage of more accelerometers in order to improve localisation accuracy.

\section{ACKNOWLEDGEMENTS}

The authors wish to thank all partners in the project (www.smarta-project.it), specifically: Datamed S.r.l., Flextronics Design S.r.l., Argonet S.r.l., Software Team S.r.l., Electron, Dipartimento di Informatica UNIMI, Dipartimento di Design of Politecnico di Milano and CoDeBri. This work was supported by a financial grant from Regione Lombardia ('Avviso pubblico per la realizzazione di progetti di ricerca industriale e sviluppo sperimentale nel settore delle Smart cities and Communities POR-FESR 2007-2013 asse 1 - Linea di intervento 1.1.1.1. azione E').

\section{REFERENCES}

[1] M. E. Tinetti, C. S. Williams, The effect of falls and fall injuries on functioning in community-dwelling older persons, The Journal of Gerontology A, Biological Sciences and Medical Sciences 53 (1998), p. M112-9.

[2] J. J. Siracuse, D. D. Odell, S. P. Gondek, S. R. Odom, E. M. Kasper, C. J. Hauser, D. W. Moorman, Health care and socioeconomic impact of falls in the elderly, The American Journal of Surgery 203 (2012) pp. 335-338.

[3] B. Najafi, K. Aminian, A. Paraschiv-Ionescu, F. Loew, C. J. Bula, P. Robert, Ambulatory system for human motion analysis using a kinematic sensor: monitoring of daily physical activity in the elderly, IEEE Transactions on Biomedical Engineering 50 (2003) pp. 711-723.

[4] M. Tarabini, B. Saggin, M. Bocciolone, D. Scaccabarozzi, M. Magni, Falls in older adults: Kinematic analyses with a crash test dummy, Proc. of the 2016 IEEE International Symposium on Medical Measurements and Applications (MeMeA), 15-18 May 2016, Benvento, Italy, pp. 1-6.

[5] G. Andreoni, F. Costa, A. Attanasio, G. Baroni, S. Muschiato, P. Nonini, A. Pagni, R. Biraghi, R. Pozzi, M. Romero, Design and ergonomics of monitoring system for elderly, Proc. of the International Conference on Digital Human Modeling and Applications in Health, Safety, Ergonomics and Risk Management, 22-27 June 2014, Heraklion, Crete, Greece, pp. 499-507.

[6] X. Yu, Approaches and principles of fall detection for elderly and patient, Proc. of the $10^{\text {th }}$ International HelathCom Conference on E-Health Networking, Applications and Services, 7-9 July 2008, Singapore, Singapore pp. 42-47.

[7] S. Chaudhuri, D. Oudejans, H. J. Thompson, G. Demiris, Realworld accuracy and use of a wearable fall detection device by older adults, Journal of the American Geriatrics Society 63 (2015) pp. 2415-2416.

[8] J. Chen, K. Kwong, D. Chang, J. Luk, R. Bajcsy, Wearable sensors for reliable fall detection, Engineering in Medicine and Biology Society (2005) pp. 3551-3554.

[9] T. Zhang, J. Wang, L. Xu, P. Liu, Fall detection by wearable sensor and one-class SVM algorithm, in: Anonymous Intelligent Computing in Signal Processing and Pattern Recognition, Springer, Berlin, Heidelberg, 2006, pp. 858-863.

[10] J. Dai, X. Bai, Z. Yang, Z. Shen, D. Xuan, PerFallD: A pervasive fall detection system using mobile phones, Proc. of the $8^{\text {th }}$ IEEE International Conference on Pervasive Computing and Communications Workshop (PERCOM Workshops), 29 March 2 April 2010, Mannheim, Germany, pp. 292-297.

[11] C. Rougier, J. Meunier, A. St-Arnaud, J. Rousseau, Monocular 3D head tracking to detect falls of elderly people, Proc. of the $28^{\text {th }}$ Annual International Conference of the IEEE, Engineering in Medicine and Biology Society 2006, EMBS'06., 30 August - 3 September 2006, New York, USA, pp. 6384-6387.

[12] G. Mastorakis, D. Makris, Fall detection system using Kinect's infrared sensor, Journal of Real-Time Image Processing 9 (2014) pp. 635-646. 
[13] E. E. Stone, M. Skubic, Evaluation of an inexpensive depth camera for passive in-home fall risk assessment, Proc. of the $5^{\text {th }}$ International Conference on Pervasive Computing Technologies for Healthcare (PervasiveHealth) and Workshops, 23-26 May 2011, Dublin, Ireland, pp. 71-77.

[14] E. E. Stone, M. Skubic, Fall detection in homes of older adults using the Microsoft Kinect, IEEE Journal of Biomedical and Health Informatics 19 (2015) pp. 290-301.

[15] J. Steward, D. Lichti, J. Chow, R. Ferber, S. Osis, Performance assessment and calibration of the Kinect 2.0 time-of-flight range camera for use in motion capture applications, FIG Working Week 2015 (2015) pp. 1-14.

[16] J. Shotton, T. Sharp, A. Kipman, A. Fitzgibbon, M. Finocchio, A. Blake, M. Cook, R. Moore, Real-time human pose recognition in parts from single depth images, Communications of the ACM 56 (2013) pp. 116-124.

[17] A Williams, D. Ganesan, A. Hanson, Aging in place: fall detection and localization in a distributed smart camera network, Proc. of the $15^{\text {th }}$ ACM International Conference on Multimedia, 25-29 September 2007, Augsburg, Germany, pp. 892-901.

[18] C. Rougier, J. Meunier, Demo: Fall detection using 3D head trajectory extracted from a single camera video sequence, Journal of Telemedicine and Telecare 11 (2005) pp. 37-42.

[19] M. Alwan, P. J. Rajendran, S. Kell, D. Mack, S. Dalal, M. Wolfe, R. Felder, A smart and passive floor-vibration based fall detector for elderly, Proc of the $2^{\text {nd }}$ International Conference on Information \& Communication Technologies, 24-28 April 2006, Damascus, Syria, pp. 1003-1007

[20] D. Litvak, Y. Zigel, I. Gannot, Fall detection of elderly through floor vibrations and sound, Proc. of the 30th Annual International Conference of the IEEE Engineering in Medicine and Biology Society, 20-25 August 2008, Vancouver, BC, Canada, pp. 46324635.

[21] Y. Zigel, D. Litvak, I. Gannot, A method for automatic fall detection of elderly people using floor vibrations and sound: Proof of concept on human mimicking doll falls, IEEE Transactions on Biomedical Engineering 56 (2009) pp. 2858-2867.

[22] M. Mubashir, L. Shao, L. Seed, A survey on fall detection: Principles and approaches, Neurocomputing 100 (2013) pp. 144152

[23] G. Igrejas, J. S. Amaral, P. J. Rodrigues, Fall detection system for elderly people: a neural network approach, Final Program and Book of Abstracts BIOSTEC 2012 (2012) pp. 154-154.

[24] C. Doukas, I. Maglogiannis, Advanced patient or elder fall detection based on movement and sound data, Proc. of the $2^{\text {nd }}$ International Conference on Pervasive Computing Technologies for Healthcare, 31 January - 2 February 2008, Tampere, Finland, pp. 103-107.
[25] D. E. Allen, Building vibrations from human activities, Concrete International 12 (1990) pp. 66-73.

[26] A. Blakeborough, M. Williams, Measurement of floor vibrations using a heel drop test. Proc. of the Institution of Civil EngineersStructures and Buildings 156, 2003, pp. 367-371.

[27] J. Tan, J. J. Eng, S. N. Robinovitch, B. Warnick, Wrist impact velocities are smaller in forward falls than backward falls from standing, Journal of Biomechanics 39 (2006) pp. 1804-1811.

[28] K. DeGoede, J. Ashton-Miller, Fall arrest strategy affects peak hand impact force in a forward fall, Journal of Biomechanics 35 (2002) pp. 843-848.

[29] C. Smeesters, W. C. Hayes, T. A. McMahon, Disturbance type and gait speed affect fall direction and impact location, Journal of Biomechanics 34 (2001) pp. 309-317.

[30] C. Asensio, G. Moschioni, M. Ruiz, M. Tarabini, M. Recuero, An intelligent thrust reverse noise detector, Noise \& Vibration Worldwide 45 (2014) pp. 13-18, DOI: https:// doi.org/10.1260/0957-4565.45.6.13.

[31] C. Asensio, G. Moschioni, M. Ruiz, M. Tarabini, M. Recuero, Implementation of a thrust reverse noise detection system for airports, Transportation Research Part D: Transport and Environment 19 (2013) pp. 42-47, DOI: https://doi.org/10.1016/j.trd.2012.12.003.

[32] M. Tarabini, G. Moschioni, C. Asensio, D. Bianchi, B. Saggin, Unattended acoustic events classification at the vicinity of airports, Applied Acoustics Volume 84 (2014) pp. 91-98. DOI: https://doi.org/10.1016/j.apacoust.2014.03.013.

[33] B. Saggin, D. Scaccabarozzi, M. Tarabini, Optimized design of suspension systems for hand-arm transmitted vibration reduction, Journal of Sound and Vibration 331 (2012) pp. 2671-2684. DOI: https://doi.org/10.1016/j.jsv.2012.01.024.

[34] M. Tarabini, B. Saggin, D. Scaccabarozzi, G. Moschioni, Hand-arm mechanical impedance in presence of unknown vibration direction, International Journal of Industrial Ergonomics 43 (2013) pp. $52-61$

[35] P. Gardonio, S. Elliott, Driving point and transfer mobility matrices for thin plates excited in flexure, NASA (1998).

[36] P. Gardonio, M. J. Brennan, Mobility and impedance methods in structural dynamics, in: F. Fahy, J. Walker (Eds.), Advanced Applications in Acoustics, Noise and Vibration, Spoon Press, London, 2004

[37] L. Gaul, S. Hurlebaus, Identification of the impact location on a plate using wavelets, Mechanical Systems and Signal Processing 12 (1998) pp. 783-795 